\title{
Crystallization kinetics of supercooled liquid Ge-Sb based on ultrafast calorimetry
}

\author{
Bin Chen, Jamo Momand, Paul A. Vermeulen and Bart J. Kooi \\ Zernike Institute for Advanced Materials, University of Groningen, Nijenborgh 4, 9747 AG \\ Groningen, The Netherlands
}

\section{Weighting data in Kissinger plot}

Figure S1 shows a Kissinger plot for all the data obtained by ultrafast DSC measurements of $\mathrm{Ge}_{7} \mathrm{Sb}_{93}$ flakes (see the red circles in Fig. S1). One can see, particularly at higher heating rates $\Phi$, that there is quite some scatter in peak temperature $\left(T_{p}\right)$ values for a single $\Phi$. This variation can be ascribed to several reasons, such as the thermal contact between the flakes and the chip sensor and the stochastic nature of crystal nucleation in the $\mathrm{Ge}_{7} \mathrm{Sb}_{93}$ film. Note that it may be expected that this stochastic nature is more apparent in case of a growthdominant PCM analyzed in the present work, and is reduced in case of nucleation-dominant PCMs like $\mathrm{Ge}_{2} \mathrm{Sb}_{2} \mathrm{Te}_{5}$ (see the blue diamonds in Figure S1). However, the data in Figure S1 clearly shows that such a difference (for the red and blue data) cannot be observed. The results with the lowest $T_{p}$ are given the most weight because it contains the highest nuclei density and of course better thermal contact between the flakes GeSb and the chip sensor. So, the dominant process in the crystallization under ultrafast DSC for GeSb alloy is then crystal growth. Therefore, for each $\Phi$, the 2 or 3 data points with lowest $T_{p}$ have been used for our analysis, as shown in Figure 2 in the main text. 


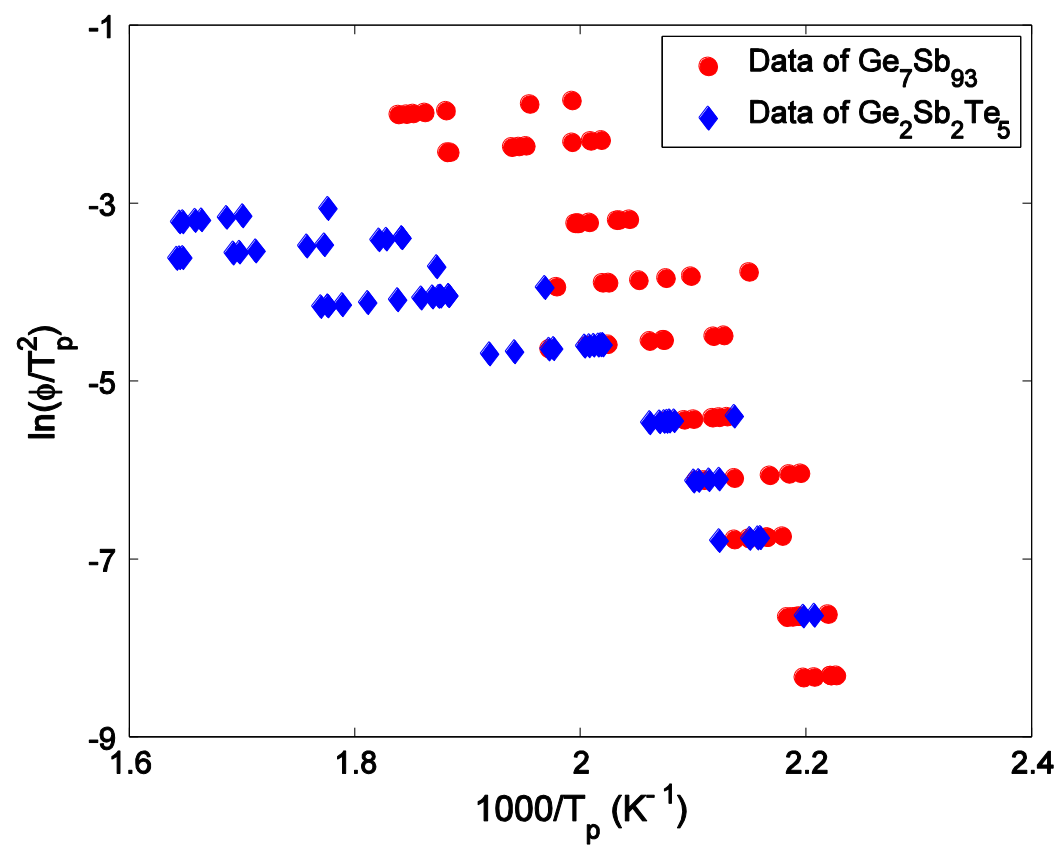

Figure S1. Kissinger plot of all the measured data of flaked $\mathrm{Ge}_{7} \mathrm{Sb}_{93}$ films and $\mathrm{Ge}_{2} \mathrm{Sb}_{2} \mathrm{Te}_{5} \mathrm{PCM}$. This figure shows that the peak temperatures $T_{p}$ of ultrafast DSC measurements on the growth dominated PCMs vary significantly, especially on the high heating rate $\Phi$ side. In our analysis the data with lowest $T_{p}$ 's (i.e., the most right 2-3 data points for each $\Phi$ ) were given to the most weight, because correspond to the flakes containing the highest nuclei density and the best thermal contact between the materials and the chip sensor. The red circles represent the data of the $G e_{7} S b_{93} P C M$, while the blue diamonds are results of the $\mathrm{Ge}_{2} \mathrm{Sb}_{2} \mathrm{Te}_{5} P C M$ s. Pronounced curvature in the Kissinger plot at higher $\Phi$ can be clearly observed in this figure, which appears clearly more pronounced for $\mathrm{Ge}_{2} \mathrm{Sb}_{2} \mathrm{Te}_{5}$.

\section{Numerical simulations via JMAK theory}

We have employed a model based on numerical calculations to fit our experimental data in the Kissinger plot, similar as was performed by Orava et al.. ${ }^{1}$ The JMAK theory is used here. ${ }^{2-5}$ To perform the numerical calculations, we consider, like in experiment, a constant $\Phi$, which we can approximate by taking many short isothermal steps. Here $0.05 \mathrm{~K}$ per step is used. Given a certain (model for the) growth rate, the JMAK theory is used to calculate the DSC traces for different $\Phi$.

In classical JMAK theory, the extended transformed space $X_{e}$ can be described as: ${ }^{2-5}$

$X_{e}=f \int_{0}^{t} I(\tau)\left[\int_{\tau}^{t} u(t) d t\right]^{n} d \tau$ 
in which $I(\tau)$ is the time-dependent nucleation rate (per unit of untransformed space) and $u(t)$ is the time-dependent growth rate of the crystals. $f$ and $n$ are shape factors related to the growth shape; i.e. for circular growth in 2 dimensions $f$ and $n$ are $\pi$ and 2, respectively, and for spherical growth in 3 dimensions $f$ and $n$ are $4 \pi / 3$ and 3, respectively. However, Orava et al. adopted that the nucleation for the $\mathrm{Ge}_{2} \mathrm{Sb}_{2} \mathrm{Te}_{5}$ (which is a nucleation dominant phasechange material) can be treated using a constant density of pre-existing nuclei (site saturation). ${ }^{1}$ It is worth noting that this assumption is justified for the (growth dominated) $\mathrm{Ge}_{7} \mathrm{Sb}_{93}$ phase-change material we analyze here, as can be demonstrated from our previous work and as will be highlighted in the next section. Therefore we can simplify the $X_{e}$ as:

$X_{e}=f N\left[\int_{0}^{t} u(t) d t\right]^{n}$

with $N$ the density of pre-existing nuclei (per unit of untransformed space).

The real transformed space $(X(t))$ can then according to the JMAK formulism be directly related to extended transformed space:

$X(t)=1-\exp \left[-X_{e}(t)\right]$

If we assume that the changing rate of enthalpy $(d H / d t)$ scales linearly with the changing rate of the real transformed space, then the DSC signal can be written as: ${ }^{6}$

DSC signal: $\propto \frac{X(T+\delta T)-X(T)}{\delta t}$

with $X(T)$ the real transformed volume at temperature $T, \delta t$ the time consumed for each isothermal step. In this case, $\delta t$ is inversely proportional to $\Phi(\delta t=\delta T / \Phi)$. Therefore, higher $\Phi$ lead to increments of (the area under) exothermic peaks in the DSC traces at higher temperatures (Figure 1 in the main text).

\section{Estimation of number density of nuclei $N$}

During fitting the data in the Kissinger plot by means of the growth models and JMAK theory, we find that the nucleation density $N$ impacts the final fitting results. For instance, the fitting fragility $m$ is shifted from 65 to 95 in the S\&M model if the $N$ is exaggeratedly lowered from $10^{16} \mathrm{~m}^{-3}$ to $10^{10} \mathrm{~m}^{-3}$. Interestingly, this change in $N$ does not affect the value of the glass 
transition temperature $T_{g}$ obtained by fitting the data in the Kissinger plot. Figure S2 shows in an Angell plot the result of this fitting with these two fixed values of $N$.

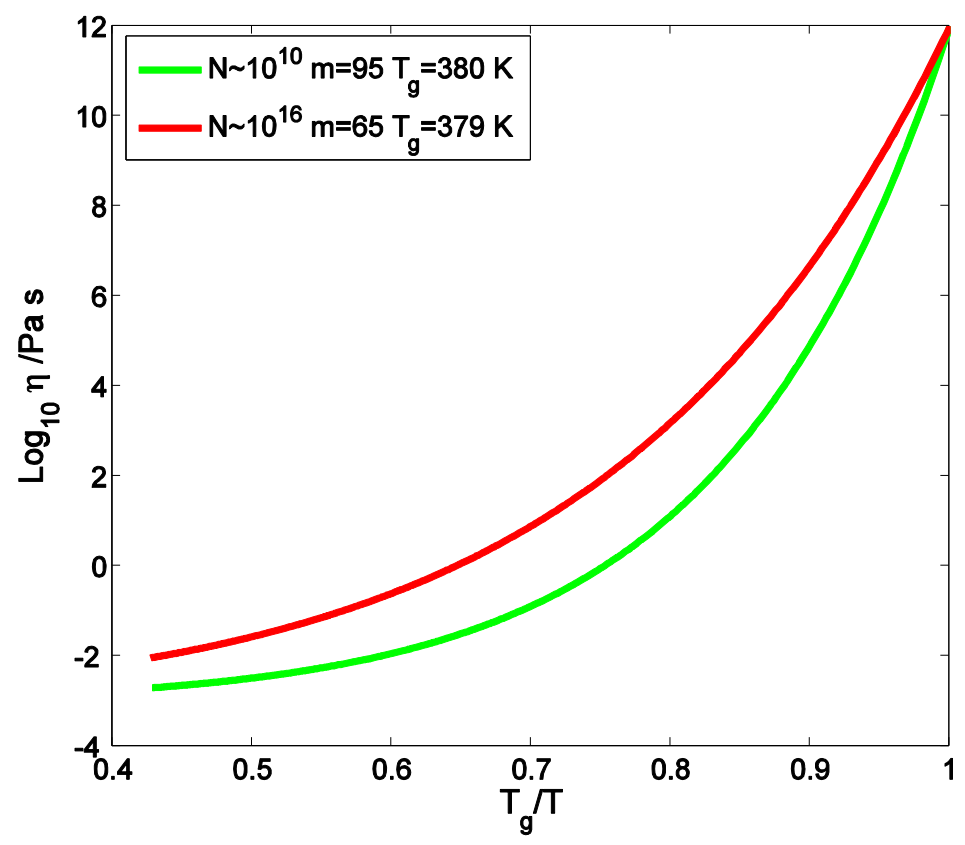

Figure S2. Angell plot with different number density of nuclei $N$. This figure displays the viscosity as function of temperature for two $N$ values, i.e. $10^{10} \mathrm{~m}^{-3}$ to $10^{16} \mathrm{~m}^{-3}$ in order to demonstrate the importance to have a reasonable estimate for $N$. While the fitted $T_{g}$ scarcely changed $(\sim 380 \mathrm{~K})$, the $\mathrm{m}$ changes remarkably from 95 to 65.

Because of the importance to have a reasonable estimate for $N$, it is required to have a reasonable approximation of the nuclei numbers during heating in the ultrafast DSC. The strength of our current approach is that we have this estimate based on our earlier work employing a high speed optical camera to monitor the nucleation and growth of crystals in the $200 \mathrm{~nm}$ thick $\mathrm{Ge}_{7} \mathrm{Sb}_{93}$ films. ${ }^{7,8}$ The phase transition of these films was investigated via isochronal heating on a ceramic heater at a $\Phi$ of $10{ }^{\circ} \mathrm{C} \min ^{-1}$. Observable nuclei develop at about $130{ }^{\circ} \mathrm{C}$. Figure $\mathrm{S} 3 \mathrm{a}$ and $\mathrm{b}$ show the change of nuclei with temperature, increasing for the area observed from 123 at $133.3{ }^{\circ} \mathrm{C}$ to 147 at $142.3{ }^{\circ} \mathrm{C}$. The number of nuclei thus only increases by $20 \%$. An approximation of $N$ is achieved as $\sim 4.1 \times 10^{14} \mathrm{~m}^{-3}$ at $142{ }^{\circ} \mathrm{C}$ when a 3dimensional growth model is adopted for the crystals and considering the thickness of this film $(200 \mathrm{~nm})$. From the video made from this measurement, it is clear that the number of nuclei hardly increase after $140{ }^{\circ} \mathrm{C}$ for this $\Phi$. After the initial nucleation below $135^{\circ} \mathrm{C}$, the dominant process of crystallization is thus growth of crystals. Moreover, in all our 
measurements of crystallization of $\mathrm{Ge}_{7} \mathrm{Sb}_{93}$ films we observed that all crystals observed in a certain area tend to have the same size. These observations thus show that the constant nuclei assumption in the numerical calculations via JMAK theory is justified.

However, in another measurement, it is found that scraping off the $\mathrm{Ge}_{7} \mathrm{Sb}_{93}$ films remarkably enlarges the possibility of nucleation as shown in Figure S4c, where areas which have been scratched (and which probably experienced compressive stress) experience earlier nucleation than the surrounding PCM under isothermal heating, resulting in a greater value for $N$. Accelerated crystallization by applying modest compressive stresses to $\mathrm{Ge}_{6} \mathrm{Sb}_{94}$ or $\mathrm{Ge}_{7} \mathrm{Sb}_{93}$ films has also been demonstrated by our previous work. ${ }^{9}$ Stress-induced crystallization of $\mathrm{Ge}_{15} \mathrm{Sb}_{85}$ has also been discussed by Shakhvorostov et al. ${ }^{10}$ Therefore, the real $N$ in the ultrafast DSC measurements must be larger than $10^{14} \mathrm{~m}^{-3}$. On the other hand, with an $N$ value larger than $10^{17} \mathrm{~m}^{-3}$ the data in the Kissinger plot in Figure 2 of the main text could not be fitted. So, the reasonable region of $N$ values is $10^{15}$ to $10^{16} \mathrm{~m}^{-3}$ in our fitting, and the one we used is $10^{16} \mathrm{~m}^{-3}$. Note this is the starting value employed in the fitting procedure using the downhill Simplex method and therefore still some minor adjustment of number density of nuclei $N$ is possible as a result of the fitting procedure. For this fitting a value of $5 \times 10^{16} \mathrm{~m}^{-3}$ is derived for $N$.
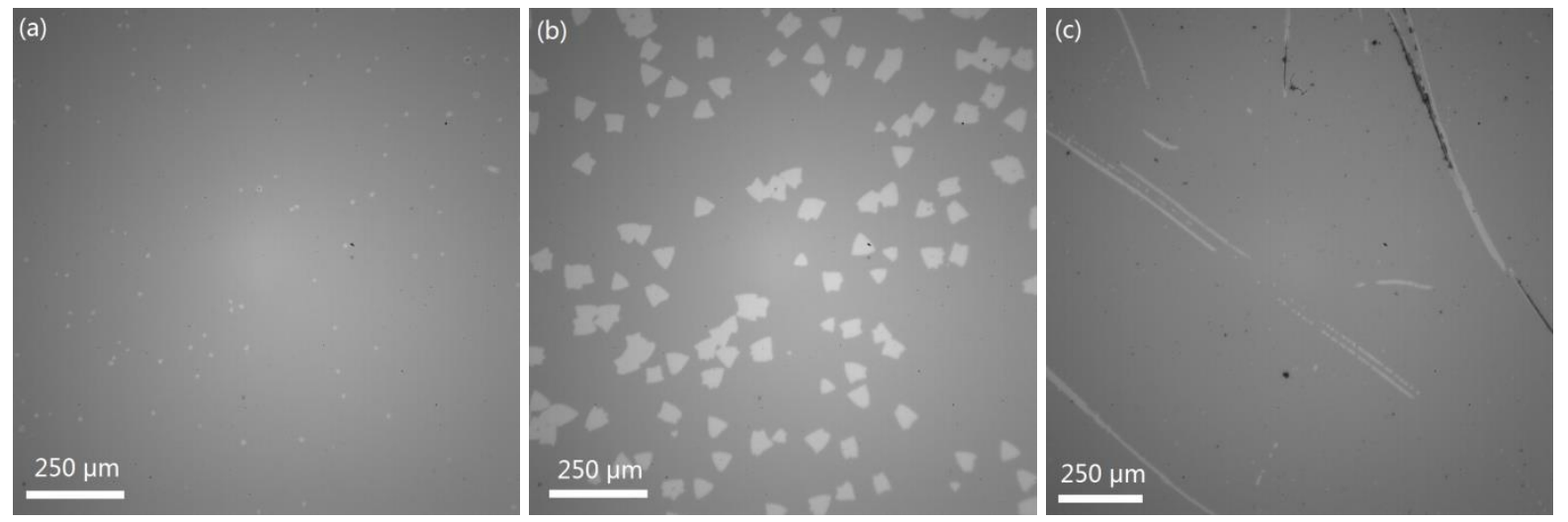

Figure S3. $\mathrm{Ge}_{7} \mathrm{Sb}_{93}$ films under isochronal heating. (a) shows the nuclei number of 123 at $133.3{ }^{\circ} \mathrm{C}$; (b) shows the number of nuclei changes to 147 at $142.3{ }^{\circ} \mathrm{C}$. From (a) and (b) the density of nuclei can be derived for both 2-dimensional $\left(8.2 \times 10^{7} \mathrm{~m}^{-2}\right)$ and 3-dimensional $\left(4.1 \times 10^{14} \mathrm{~m}^{-3}\right)$ crystal growth for JMAK theory at $142{ }^{\circ} \mathrm{C}$, as the thickness of the film is $200 \mathrm{~nm}$. (c) shows the pressure-induced nucleation on $\mathrm{Ge}_{7} \mathrm{Sb}_{93}$ films. (c) The scratches made by a hair nucleate earlier than the surrounding materials under isothermal heating at $125^{\circ} \mathrm{C}$, with an obviously higher nuclei density. 
In the fittings described above, the crystal growth model used for JMAK theory is spherical growth in 3 dimensions. However, the PCM films studied here only have a thickness of 200 $\mathrm{nm}$, but the crystals can easily grow to lateral sizes larger than $1 \mu \mathrm{m}$ under the $\Phi$ of $10 \mathrm{~K} / \mathrm{min}$, shown in Figure S3. For the lower $\Phi$ employed in ultrafast DSC, it is highly possible that the 3-dimensional growth model adopted in JMAK theory does not hold. Therefore it is necessary to check the accuracy of the fittings in this article when we consider instead 2-dimensional crystal growth (of $200 \mathrm{~nm}$ thick cylinders) in the JMAK theory and in the fitting procedure.

For a 2-dimensional growth model, the density of nuclei from Figure S3 can be readily calculated as $8.2 \times 10^{7} \mathrm{~m}^{-2}$ at $142{ }^{\circ} \mathrm{C}$. With the stress induced nucleation in consideration, we take $N$ of $\sim 10^{10} \mathrm{~m}^{-2}$ as the starting point to fit the Kissinger plot for both the S\&M model and the C\&G models. Taking the crystal shape as $200 \mathrm{~nm}$ thick cylinders (although the crystals appear to be triangles, but shape does not significantly affect the fitting) in JMAK theory, parameters obtained here are $T_{g}=380 \mathrm{~K}, m=66, N \sim 8 \times 10^{10} \mathrm{~m}^{-2}$, with $\mathrm{R}^{2}=0.923$ for the $\mathrm{S} \& \mathrm{M}$ model (parameters for spherical growth in 3 dimensions were $T_{g}=379 \mathrm{~K}, m=65, N \sim 5 \times 10^{16} \mathrm{~m}^{-}$ ${ }^{3}$, with $\left.\mathrm{R}^{2}=0.924\right)$. For the $\mathrm{C} \& \mathrm{G}$ model, the obtained fitting parameters slightly change (from $B=119.2, C=1.5, T_{0}=443 \mathrm{~K}$, with $\mathrm{R}^{2}$ of 0.937 ) to $B=80, C=1.06, T_{0}=448 \mathrm{~K}$, resulting in the same $m$ of 86 . Figure $\mathrm{S} 4$ shows the Kissinger plot for the fitting of the 2-dimensional crystal growth for the $S \& M$ and the $C \& G$ models. The derived growth rates of the $C \& G$ model are still 2-3 orders of magnitude higher than that of the independent data. Therefore, we can draw the firm conclusion that no significant differences occur when the crystal growth model changes from 3-dimensional to 2-dimensional, indicating the reliability of the fitting in the main text. 


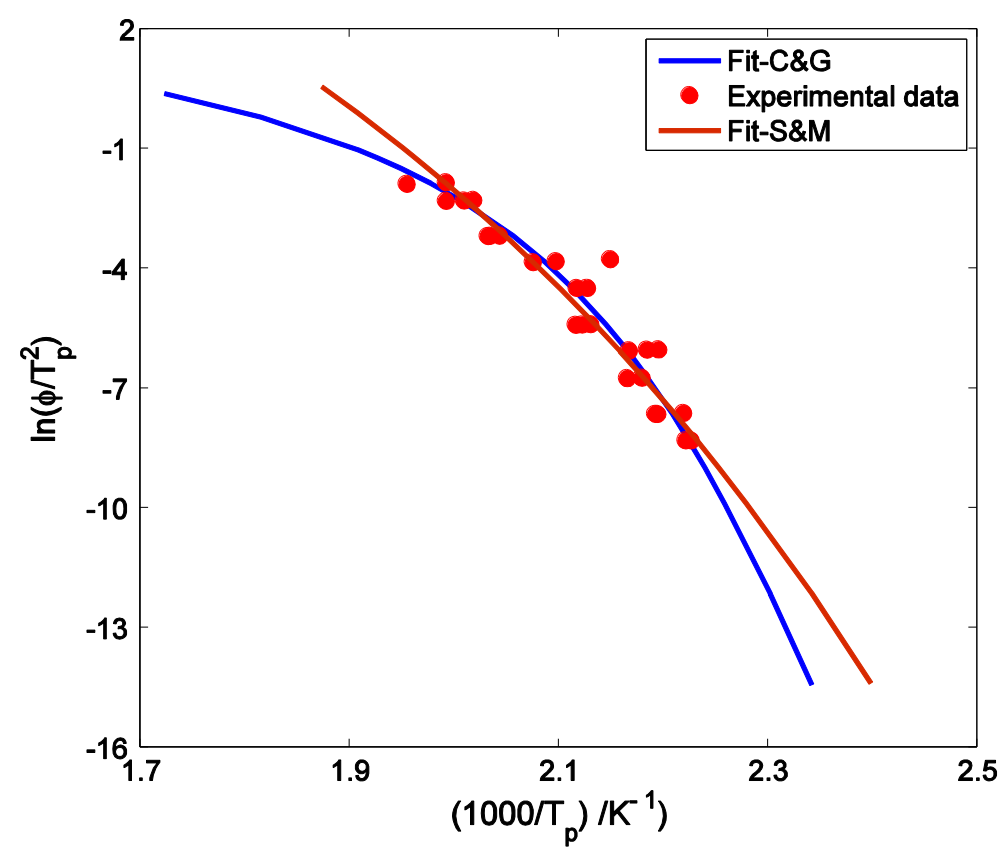

Figure S4. Kissinger plot with 2-dimensinal fittings. Similar to Figure2 in the main text, the S\&M model and the $C \& G$ model are adopted to fit the experimental data in Kissinger plot. 2-dimensional crystal growth is employed in the JMAK theory instead the 3-dimensional crystal growth in the main text. Reasonable fits can be observed on this figure based on both models, giving similar fitting parameters as the 3-dimensional crystal growth. Therefore the properties obtained from fitting maintain the same as in the main text, indicating negligible influence of the dimensionality of the crystal growth in this fitting.

\section{Effect of the $A$ parameter in the Cohen \& Grest expression for determining the growth rate}

In Figure S5, the influence of the value of $A$ in the C\&G model (see Equation 5 in the main article) on the growth rate is shown. The blue curve shows the growth rate derived from the Stokes-Einstein equation, identical to the one in Figure 4 in the main text, leading to a maximum growth rate $\left(U_{m}\right)$ of $17 \mathrm{~m} \mathrm{~s}^{-1}$. The green curve in this figure is derived when $A$ is set to the 0 , which is the value employed in the fitting of the Kissinger plot (Figure 2 in the main text). A $U_{m}$ of $0.17 \mathrm{~m} \mathrm{~s}^{-1}$ is obtained here. Then, the value of $A$ is adapted in order to match the independent data from Ref. 7. Then $A$ becomes -0.4 and the corresponding growth rate curve is the khaki one in Figure S5. It is nicely shown that this modeled growth rate matches well with the independent data in the limited measurement region of the ultrafast DSC which is between 450 and $505 \mathrm{~K}$, but still leads to a poor fit for temperatures below 450 
K. Moreover, this $A$ value results in a unacceptable low value of $U_{m}\left(0.07 \mathrm{~m} \mathrm{~s}^{-1}\right)$. From the original equation ( 5 in the main article) and from the graph it is obvious that the effect of $A$ in the $C \& G$ model is that it only changes the absolute value of the of the growth rate and thus only results in a vertical shift of the curves in Figure S5. The present results thus strongly suggest that this C\&G model is not suitable to describe the growth rate of PCMs, at least for the growth dominated GeSb alloy. However, quite similar discrepancies between modelled and experimental results are observable for the nucleation dominated $\mathrm{Ge}_{2} \mathrm{Sb}_{2} \mathrm{Te}_{5}$ alloy in Figure 1 of Ref. 11. Therefore, indeed, it appears justified to question the appropriateness of the $\mathrm{C} \& \mathrm{G}$ model for PCMs in general.

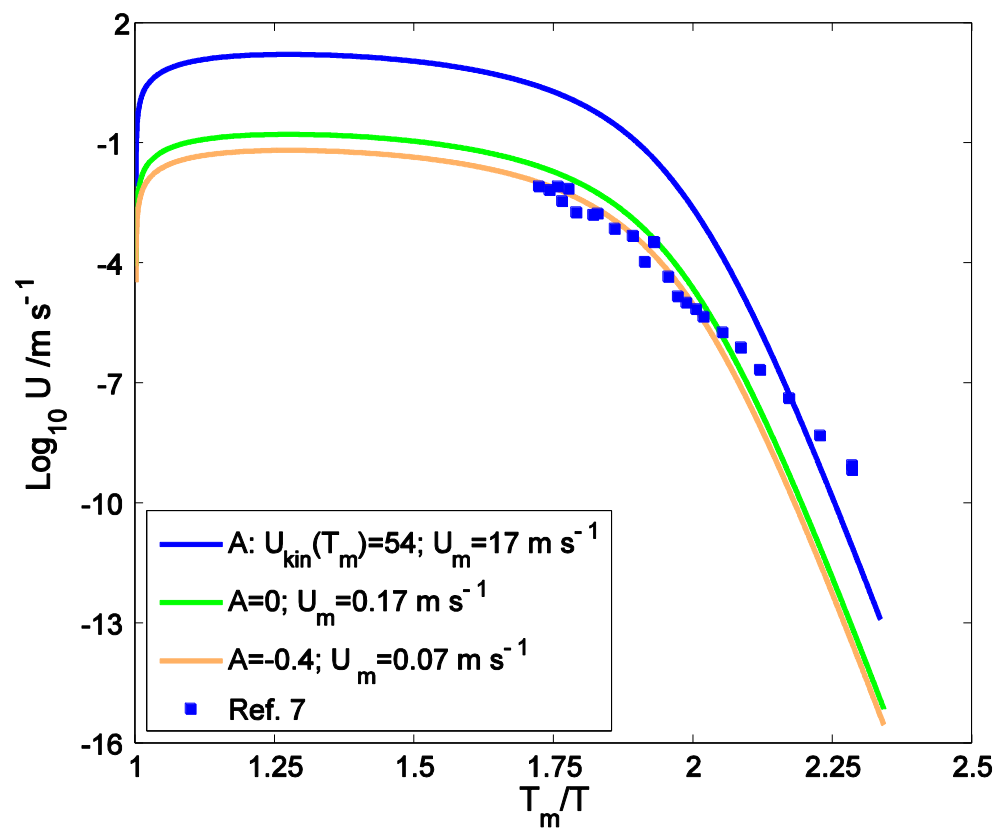

Figure S5. Growth rate from the $C \& G$ model for different values of parameter A. The green curve is identical to the one shown in the Figure 4 in the main text, which leads to a maximum growth rate value $\left(U_{m}\right)$ of $17 \mathrm{~m} \mathrm{~s}^{-1}$. The khaki curve relates to the value $A=0$, which is the one used for fitting in the Kissinger plot. $A U_{m}$ of $0.17 \mathrm{~m} \mathrm{~s}^{-1}$ is obtained. Then the value of $A$ is set to make the modeled growth rate match to the independent data. A=-0.4, the green curve is obtained as the growth rate. Although good match to the data can be seen, an unacceptable value of $U_{m}\left(0.07 \mathrm{~m} \mathrm{~s}^{-1}\right)$ is derived here. 


\section{References}

1. Orava, J.; Greer, A. L.; Gholipour, B.; Hewak, D. W.; Smith, C. E. Nat. Mater. 2012, 11, 279-283.

2. Avrami, M. J. Chem. Phys. 1939, 7, 1103-1112.

3. Avrami, M. J. Chem. Phys. 1940, 8, 212-224.

4. Kooi, B. J. Phys. Rev. B 2004, 70, 224108.

5. Kooi, B. J. Phys. Rev. B 2006, 73, 054103.

6. Kelton, K. F. Mater. Sci. Eng. A 1997, 226-228, 142-150.

7. Eising, G.; Van Damme, T.; Kooi, B. J. Cryst. Growth Des. 2014, 14, 3392-3397.

8. Eising, G.; Niebuur, B. J.; Pauza, A.; Kooi, B. J. Adv. Funct. Mater. 2014, 24, 1687-1694.

9. Eising, G.; Pauza, A.; Kooi, B. J. Cryst. Growth Des. 2012, 13, 220-225.

10. Shakhvorostov, D.; Nistor, R. A.; Krusin-Elbaum, L.; Martyna, G. J.; Newns, D. M.; Elmegreen, B. G.; Liu, X.; Hughes, Z. E.; Paul, S.; Cabral, C.; Raoux, S.; Shrekenhamer, D. B.; Basov, D. N.; Song, Y.; Müser, M. H. Proc. Natl. Acad. Sci. 2009, 106, 10907-10911.

11. Wuttig, M.; Salinga, M. Nat. Mater. 2012, 11, 270-271. 